\title{
Neuropsychobiology 1988;20:218
}

\section{Author Index}

Addy, O. 152 Albanesi, G. 78 Anderer, P. 51 Aranko, K. 82 Arriaga, F. 23 Asenbaum, S. 51 Azorin, J.-M. 67

Balbi, D. 120 Baron, P.G. 74 Bartel, P. 212 Battegay, R. 28 Beneroso, N. 136 Berger, M. 36 Bet, L. 74 Blom, M. 212 Bondiolotti, G.P. 113 Born, J. 145 Bossert, S. 36 Bottini, G. 74 Bourin, M. 136,187 Braconnier, A. 1 Brambilla, F. 113 Bresolin, N. 74 Buchsbaum, M.S. 15,62 Bursztejn, C. 1

Cairoli, S. 120 Chachkes, M. 57 Chachkes, O. 57 Chen, Y.-Y. 132 Chrisomalis, L. 169 Classen,W. 87

Deecke, L. 51 DeMet, E. 62 DePalma, D. 120 Dimpfel, W. 164 Dreux, C. 1 Dugovic, C. 23

Earle, J.B. 96

Fähndrich, E. 141 Faravelli, C. 78 
Fehm, H.L. 145 Feliciano, M. 169 Ferrari, P. 1 Fieve, R.R. 169 File, S.E. 82,205 Filippi, U. 120 Foliart, R.H. 152 Fritze, J. 87

Ganry, H. 187 Garreau, M. 126 Gastpar, M. 28 Goodnick, P.J. 169 Green, M. 15 Grillo, W. 113 Guardiola-Lemaitre, B.J.

Haier, R. 15 Haier, R.J. 62 Haimart, M. 1 Halaris, A. 152 Haug, H.-J. 141 Hitzler, V. 145 Hugdahl, K. 194

Itoh, A. 174 Iwata, T. 174

Jansen, A.A.I. 91 Jerushalmy, Z. 57 Joanny, P. 67 Joffe, R.T. 12 Junker, M. 36

Karege, F. 67 Kasahara, Y. 174 Kasall, H. 51 Kassir, S. 169 Kergueris, M.F. 136 Kerkhofs, M. 178 Klerk Sommers, de 212 Kogawa, S. 174 Krieg, J.-C. 36 
acroix, B.P. 178 Larousse, C. 136 Latina, A. 113 Launay, J.M. 1 Lee, S. 15

Le Normand, Y. 136 Levitt, A. J. 12 Lovrek, A. 158 Luong, C. 1

Maggioni, M. 113 Magni, G. 126 Mark, M. 57 Masturzo, P. 120 Meltzer, H.L. 169205 Mendlewicz, J. 178 Meola, G. 74 Meyden, C. van der 212 Miller, M.H. 43 Modai, I. 57 Mohl, W. 51 Monza, G.C. 74 Murialdo, G. 120

Nickel,,B. 164

Ohta, T. 174 Okada, T. 174 Orofiamma, B. 126 Ozaki, N. 174

Palminteri, R. 126 Pasques-Bondoux, D. 1 Pauschinger, P. 145 Petsche, H. 158 Pezzoli, G. 74 Picotti, G.B. 113 Pịetrowsky, R. 145 Prịnguey, D. 67

Randolph, C. 43 Rappelsberger, P. 158 Ribeyrol, M. 136 
Saletu, B. 51 Sanna, F. 113 Saran, A. 152 Scarlato, G. 74 Scarpini, E. 74 Schreiber, W. 36 Schubert, D.S.P. 152 Sciascia, A. 113 Sessarego, A. 78 Simhandl, Ch. 158 Slangen, J.L 91 Somenzini, G. 120 Spiss, Ch. 51 Spüler, M. 164 Stanus, E.L. 178 Suarez, K. 132 Szypula, D. 169

Tabuteau, F. 1 Tang, S.W. 15 Thau, K. 158 Tissot, R. 67 Topitz, A. 158 Tosca, P. 120 Tyano, S. 57

Valewski, A. 57 Vallar, G. 74 Valli, M. 67 Verbaten, M.N. 91

Wauquier, A. 23 Wolner, E. $51 \mathrm{Wu}$, J. 62 Wu, J.C. 15

Yu, J.-H. 132

Zeitlhofer, J. 51 Zerbi, F. 120 Zerssen, D. von 36 Zmilacher, K. 28 